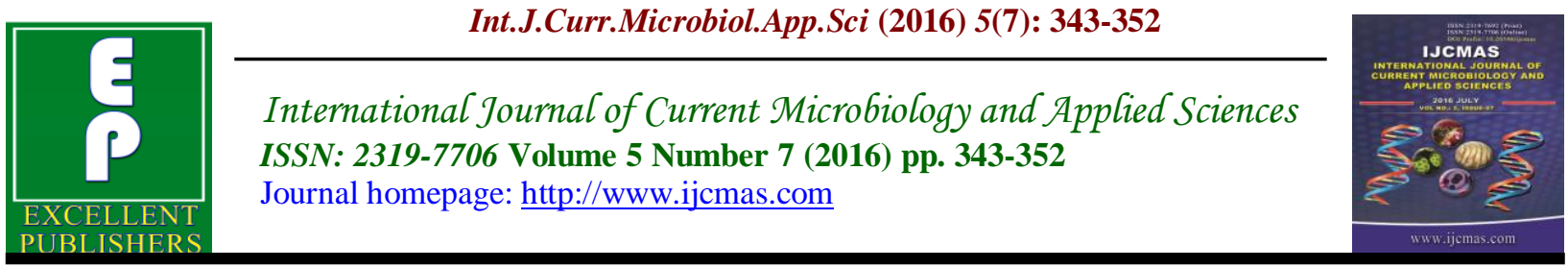

Original Research Article

http://dx.doi.org/10.20546/ijcmas.2016.507.037

\title{
The spectrum of Different Pathogens causing Ear Infections and its Antimicrobial Sensitivity Patterns in Patients from Gurgaon, Haryana state, India
}

Rishabh Rajput*
Department of Microbiology and Molecular Biology, Modern Diagnostic and
Research Centre, Gurgaon, Haryana, India
*Corresponding author

\section{Introduction}

The Ear is a vital sensory organ which is dependable for hearing and also maintaining balance, it's separated into the outer, middle and inner ear with the outer and middle regions being most vulnerable to injury and infections. Ear infection (medically termed Otitis Media) generally refers to an infection of the middle part of the ear that lies behind the eardrum. It is commendable to note that ear infections are a very common problem worldwide (Richard et al., 1998).
About 65-330 million people suffer from ear infection worldwide and $60 \%$ of them had significant hearing impairment. The discharging ear is a common problem in tropics. It is seen in all age groups but is more common in infants and children. It's falling incidence during and after an adolescent stage is the result of the growth and development of the pharynx. But still it remains one of the major problems of adults attending the Ear, Nose and Throat (ENT) clinics (Raakhee et al., 2014). 
Chronic Suppurative Otitis Media (CSOM) is chronic inflammation of the middle ear, which affects the tympanic membrane, middle ear mucosa and other middle ear structures. Clinically, CSOM presents with ear discharge and conductive deafness (Iqbal et al., 2009).

CSOM is the one of the most frequent problems related to ear in developing and developed nations if left untreated causing more severe loss of hearing. It is characterized by persistent otorrhea for more than 6-12 weeks, through the perforated tympanic membrane, usually resulting from earlier acute infection (Kristo et al., 2011).

The infection is due to the ear cleft, resultant in ear discharge (Srivastava et al., 2011). The WHO defines CSOM as 'otorrhea through a perforated tympanic membrane present for at least two weeks (WHO, 1986).

Most commonly isolated aerobic bacteria in ear infections are Staphylococcus aureus, Streptococcus pyogenes, Pseudomonas aeruginosa, Escherichia coli, Proteus spp., Klebsiella spp.

Fungal infection of the middle ear and external auditory meatus are common as fungi thrive well in moist pus and mostly isolated fungi are Aspergillus species and Candida species. But the type of organism isolated varies between the geographical area and other factors (Srivastava et al., 2011). CSOM is a global problem and affects all ages but common in children younger than 7 years due to horizontal, wider and short Eustachian tube (Haraldsson et al., 2004). The normally occurring symptoms are ear discharge, deafness, itching, pain and every so often fever. If it is left untreated complications like loss of hearing, post aural swelling and post aural sinus may occur (Oguntibeju, 2003).
CSOM has received considerable attention, not only because of its high incidence and chronicity but also because of issues such as bacterial resistance and ototoxicity with both topical and systemic antibiotics (Haynes, 2011).

Since CSOM is a disease which can cause major morbidity, knowledge of pathogens responsible can assist in the selection of the most appropriate treatment regimen and can reduce complications that may require surgical treatment. This study would be the first study in this area that provides original data about the pathogen antibiotic sensitivity profile which is likely to assist physicians in empirical therapy and setting therapeutic protocols.

\section{Materials and Methods}

\section{Study Design, period and area}

This prospective study was conducted from discharging ears specimens submitted for aerobic culture and sensitivity during the period of 6 months (November 2015 - April 2016) referred to Department of Microbiology \& Molecular Biology at Modern Diagnostic \& Research Centre, Gurgaon, Haryana -India.

\section{Collection and transport of specimen}

Samples of patients having discharging ear were collected with the help of sterile cotton swab in screw capped polypropylene tubes (Hi-Media) from all age groups using aseptic techniques and transported immediately to the microbiology laboratory.

\section{Microscopic Examination (Gram stain)}

The smear was prepared as per standard procedures for gram staining and examined 
for the presence of pus cells, bacteria and/or fungal elements. The result of the gram stain was informed to the ENT specialist within 2 hours of the collection of the swab in the department.

\section{Culture and identification}

Ear swabs were cultured on sheep blood agar (Biomerieux) \& Mac Conkey agar (HiMedia) with sterilized nichrome loop. All cases showing growth on culture media after 24 hours of incubation at $37^{\circ} \mathrm{C}$ were identified using colony morphology, gram staining and key biochemical battery of tests with standard procedures.

\section{Quality Control}

The bacterial suspension was prepared and was adjusted to a $0.5 \mathrm{McF}$ arland solution (Hi-Media). American Type Culture Collection (ATCC) standard reference strains (Pseudomonas aeruginosa ATCC27853, Staphylococcus aureus ATCC 25923, Escherichia coli ATCC-25922 were used as a quality control throughout the study for culture and antimicrobial susceptibility testing. Antibiotic and media quality control was performed on receiving of each lot and on a weekly basis. The antibiotic failed in new lot verification process was rejected.

\section{Antibiotic Sensitivity testing}

The organism was processed for antibiotic sensitivity testing by using Kirby-Bauer disc diffusion method on Mueller-Hinton agar using selected antibiotics panel according to Clinical and Laboratory Standard Institute (CLSI., 2015).

\section{Antibiotic sensitivity pattern for bacterial} isolates - Amoxicillin-clavulanate, piperacillin-tazobactam, cefepime, ceftriaxone, cefuroxime, ceftazidime, cefixime, imipenem, gentamicin, amikacin, tetracycline, ciprofloxacin, levofloxacin, ofloxacin \& cotrimoxazole were tested against Enterobacteriaceae. piperacillintazobactam, ticarcillin-clavulanate, ceftazidime, cefepime, aztreonam, imipenem, colistin, polymyxin B, gentamicin, amikacin, ciprofloxacin, levofloxacin \& ofloxacin were tested against Pseudomonas aeruginosa. Penicillin, vancomycin (E-Test), teicoplanin, gentamicin, amikacin, erythromycin, clindamycin, tetracycline, ciprofloxacin, levofloxacin, ofloxacin, cotrimoxazole, chloramphenicol, linezolid \& cefoxitin were tested against Staphylococcus spp. Ampicillin, penicillin, ofloxacin, vancomycin, teicoplanin, erythromycin, tetracycline, ciprofloxacin, levofloxacin, chloramphenicol, linezolid \& high-level gentamicin were tested against Enterococcus spp. Penicillin, vancomycin, erythromycin, azithromycin, tetracycline, levofloxacin, ofloxacin, cotrimoxazole, chloramphenicol, clindamycin \& linezolid were tested against Streptococcus pneumoniae. Penicillin, Ampicillin, cefepime, ceftriaxone, vancomycin, erythromycin, azithromycin, tetracycline, levofloxacin, ofloxacin, chloramphenicol, clindamycin and linezolid were tested against Streptococcus pyogenes. Antifungal sensitivity was not included in the study.

\section{Results and Discussion}

In this study, a total of 295 patients having ear discharge \& suspected of otitis media were included in this study. Of them, 160 (54.2\%) were males and 135 (45.8\%) were female patients with the ratio of 1.19:1. Overall, $194(65.8 \%)$ of patients with ear infection had culture positive. The proportion of ear infection with positive culture was $110(56.70 \%)$ in males \& 84 $(43.30 \%)$ in females with the ratio of 1.31:1. (Table 1) 
The majority of the patients who had ear discharge were between $<10$ years of age. This was followed by the age group between $21-30,11-20,31-40,41-50$ and $>50$. Of them, the patients who had an ear infection with the positive culture were from age group was 31-40, 41-50, <10, 21-30, >50, 11-20. (Table 2).

Out of 194 patients, there were 199 isolates. One eighty-nine patients $(64.1 \%)$ had an infection from the single organism, five patients $(1.7 \%)$ had an infection with two organisms and remaining one hundred one $(34.2 \%)$ had a sterile culture with no organisms isolated. (Table 3 ).

Of 199 isolates, $181(92.0 \%)$ were bacteria and $18(9.0 \%)$ were fungal isolates. Gram negative bacteria were isolated more frequently as compare to gram positive (47.24 and $43.72 \%$ respectively). The frequency of gram positive bacterial isolates was Staphylococcus aureus (37.2\%), Streptococcus pyogenes (3.0\%), Streptococcus pneumoniae (2.5\%) and Enterococcus spp. whereas the frequency of gram negative bacterial isolates was Pseudomonas aeruginosa (34.2\%) followed by Escherichia coli (4.0\%), Proteus mirabilis (4.0\%), Klebsiella pneumoniae (2.5\%) and Enterobacter aerogenes (2.0\%). The most frequent fungal isolates were Aspergillus spp. (5.0\%) followed by Candida spp. (4.0\%). (Table 4)

Resistance pattern of gram negative bacterial isolates was Pseudomonas aeruginosa (2.9-17.4\%), Escherichia coli (12.5-37.5\%), Enterobacter aerogenes (025.0), Proteus mirabilis (12.5-75.0\%) and Klebsiella pneumoniae (20.0-60.0\%) (Table 5).

Resistance pattern of gram positive isolates to the antibiotics tested was Staphylococcus aureus (1.4-93.2 \%), Streptococcus pyogenes (16.7-50.0\%), Streptococcus pneumoniae (20.0-40.0 \%), Enterococcus spp (0-50\%). (Table 6)

The present study would be the first study that attempts to isolate and characterize the probable pathogens among ear infections from the patients of all age groups and their antibiotic sensitivity profile from Gurgaon, Haryana State.

Ear infection is the most frequent disease for patients to visit clinician and take antibiotics (Grevers., 2010). The most frequent symptoms of ear infections are ear discharge (otorrhea), ear pain (otalgia), hearing loss, vertigo, and tinnitus (Cheng et al., 1997). In our study male $(54.2 \%)$ predominance was higher than female $(45.8 \%)$. This observation was parallel with the finding of the few authors (Kumar et al., 2011; Lodhi et al., 2010; Yousuf et al., 2011).

In this study, out of 194 patients with positive culture report, there were 199 isolates. The analysis revealed that 189 $(64.1 \%)$ had an infection from the single organism, 5I (1.7\%) had an infection with two organisms and remaining 101 (34.2\%) had a sterile culture with no organism isolated. Matching figures reported by other authors vary significantly. Aslam et al., 2004 from Pakistan, in their study on 142 samples revealed that $76 \%$ of them were pure and $23.9 \%$ were mixed culture and only $2.1 \%$ fungi whereas Poorey et al., 2002 from India, in their study on 100 samples found pure growth from 82, mixed growth from 10 and no growth in 8 samples. The difference in results of a variety of authors could have been due to a difference in the patient population studies and geographical variations.

The clinical findings of patients with otitis media occurrence and peak age prevalence (mostly in children younger than 10 years of age) in our study are similar to findings of 
previous studies done in Ethiopia and other developing countries (Melaku et al., 1999; Tobhi et al., 2006).

The high incidence rate in children is may be due to numerous reasons as young children and infants may have low resistance and also because of the relatively short Eustachian tube. Due to the short Eustachian tube, infected material from the nose, adenoids and sinuses passes more readily along the Eustachian tube to the tympanic cavity, particularly during coughing, sneezing, vomiting and forced feeding commonly practiced in our environment with the child's nose blocked, while being held head down and half prone (Nwabuisi et $a l ., 2002)$. It may be attributed to the fact that they are more prone to upper respiratory tract infection. Poor hygiene and unorthodox approach to the treatment like unconventional ear drops and concoctions such as oil and honey into the middle ear may initiate the proliferation of opportunistic pathogens leading to the blockage of the Eustachian tube (Parkash et al., 2004).

In the present study Staphylococcus aureus was found to be most predominant organism $37.2 \%$ followed by Pseudomonas aeruginosa (34.7\%), Proteus mirabilis (4.0\%) and Escherichia coli (4.0\%). Higher prevalence of Staphylococcus aureus in this is also same as other author having $54 \%$ of Staphylococcus aureus (Park et al., 2008). Similar findings have been observed in Ireland (Mubassabi., 2007), Pakistan (Arshad et al., 2004)., and Greece (Bardanis al., 2003)., which reported that $P$. aeruginosa and $S$. aureus are the most common organism isolated from the patients. The reason behind S.aureus to be most prevalent organism might be because it is an opportunistic pathogen and normal flora of the skin, but when it enters the human body it causes infection to tissue and mucous membrane (Alo et al., 2012).

The prevalence of $K$. pneumoniae and $E$. coli in this study was 2.5 and $4.0 \%$ respectively. Likewise, 3.4 and $2.4 \%$ of $K$. pneumoniae and E. coli was, respectively reported in studies from Ethiopia (Wasihun et al., 2015). Moreover, a study in India reported 8 and $4 \%$ of $K$. pneumoniae and $E$. coli, respectively through the proportion varies (Prakash et al., 2013). Isolation of fecal bacteria like $K$. pneumoniae and $E$. coli might indicate that individuals were at risk of infection due to poor hygiene conditions.

P. aeruginosa showed sensitivity to Imipenem (S-100\%), Piperacillin/ tazobactam (S- 100\%), Ceftazidime (S97.1\%, R- 2.9\%), Cefepime (97.1\%, R2.9\%), Amikacin (S-88.4\%, R-11.6\%), Gentamicin (S- 85.5\%, I- 2.9\%, R- 11.6\%), Aztreonam (S-85.5\%, I-10.1\%, R-4.3\%), Ciprofloxacin (S- 82.6\%, R- 17.4\%), Levofloxacin (S- 82.6\%, R- 17.4\%), Ofloxacin (S- 81.2\%, I-2.9\%, R-15.9\%) \& Ticarcillin/Clavulanate (S-78.3\%, I-15.9\%, R-5.8\%). The antimicrobial pattern of Imipenem, Ciprofloxacin, Ceftazidime, and Levofloxacin is in agreement with the study conducted in by Moorthy et al., 2013 Whereas the pattern of Amikacin \& Gentamicin is in agreement with the study conducted by AHC Loy et al., 2002.

$S$. aureus showed sensitivity to Vancomycin (S-100\%), Teicoplanin(S-100\%), Linezolid (S-100\%), Chloramphenicol (S-97.3\%, R2.7\%), Amikacin (S-97.3\%, I-1.4\%, R1.4\%), Tetracycline (S-91.9\%, R-8.1\%), Gentamicin (S-85.1\%, I-12.2\%, R-2.7\%), Clindamycin (S-78.4\%, R-21.6\%), Cefoxitin (S-75.7\%, R-24.3\%), Erythromycin (S68.9\%,I-9.5\%,R-21.6\%), Levofloxacin (S40.5\%,I-33.8\%,R-25.7\%), Cotrimoxazole (S-40.5\%,R-59.5\%), Ciprofloxacin (S- 
25.7\%, I-14.9\%, R-59.5\%), Ofloxacin (S$25.7 \%$, I- $14.9 \%, \mathrm{R}-59.5 \%$ ), \& penicillin (S$6.8 \%$, R-93.2\%). The antimicrobial pattern of vancomycin, Cefoxitin, Amikacin \& Gentamicin is in agreement with the study conducted in by Nazir et al., 2014.

When the results of various workers were compared, it became obvious that the profile of pathogens and antibiotic sensitivity patterns causing ear infections has been changing from time to time. The probable reasons for this variation could be contributed to antimicrobial resistance profile of bacteria varies among the population because of difference in geography, local antimicrobial prescribing practices and prevalence of resistant bacterial strains.

Table.1 Distribution of ear infection in relation to Gender

\begin{tabular}{|c|c|c|c|c|}
\hline Gender & $\begin{array}{c}\text { No of samples } \\
\text { received } \\
(\mathrm{n}=295)\end{array}$ & Percentage & $\begin{array}{c}\text { Number of } \\
\text { positive } \\
\text { Cases }(n=194)\end{array}$ & Percentage \\
\hline Male & 160 & 54.2 & 110 & 68.80 \\
\hline Female & 135 & 45.8 & 84 & 62.20 \\
\hline
\end{tabular}

Table. 2 Age and Sex wise distribution of positive cases

\begin{tabular}{|l|c|c|c|c|c|c|c|}
\hline \multirow{2}{*}{$\begin{array}{c}\text { Age } \\
\text { Years })\end{array}$} & \multicolumn{2}{|c|}{ Male } & \multicolumn{2}{c|}{ Female } & \multicolumn{2}{|c|}{ Total } & \\
\cline { 2 - 8 } & $\begin{array}{c}\text { Total } \\
\text { Received }\end{array}$ & $\begin{array}{c}\text { Positive } \\
(\boldsymbol{\%})\end{array}$ & $\begin{array}{c}\text { Total } \\
\text { Received }\end{array}$ & $\begin{array}{c}\text { Positive } \\
(\boldsymbol{\%})\end{array}$ & Total & Positive & $\%$ \\
\hline$<10$ & 50 & $34(68.0)$ & 34 & $22(64.7)$ & 84 & 56 & 66.7 \\
\hline $11-20$ & 27 & $16(59.3)$ & 20 & $9(45.0)$ & 47 & 25 & 53.2 \\
\hline $21-30$ & 47 & $32(68.1)$ & 30 & $19(63.3)$ & 77 & 51 & 66.2 \\
\hline $31-40$ & 21 & $17(81.0)$ & 23 & $17(73.9)$ & 44 & 34 & 77.3 \\
\hline $41-50$ & 8 & $6(75.0)$ & 15 & $10(66.7)$ & 23 & 16 & 69.6 \\
\hline$>50$ & 7 & $5(71.4)$ & 13 & $7(53.8)$ & 20 & 12 & 60.0 \\
\hline Total & $\mathbf{1 6 0}$ & $\mathbf{1 1 0}(\mathbf{6 8 . 8})$ & $\mathbf{1 3 5}$ & $\mathbf{8 4}(\mathbf{6 2 . 2})$ & $\mathbf{2 9 5}$ & $\mathbf{1 9 4}$ & $\mathbf{6 5 . 7}$ \\
\hline
\end{tabular}

Table.3 Number of Isolates

\begin{tabular}{|c|c|c|}
\hline $\begin{array}{c}\text { Number of } \\
\text { Isolates }\end{array}$ & $\begin{array}{c}\text { Number of } \\
\text { patients }\end{array}$ & Percentage \\
\hline 0 & 101 & 34.2 \\
\hline 1 & 189 & 64.1 \\
\hline 2 & 05 & 1.7 \\
\hline
\end{tabular}


Table.4 Microbiological profile of pathogens isolated from ear swabs ( $\mathrm{n}=199)$

\begin{tabular}{|c|c|c|c|}
\hline Type of Isolate & Type of Organism & $\begin{array}{l}\text { Frequency of } \\
\text { Isolates }\end{array}$ & $\begin{array}{c}\% \text { of Patients } \\
\text { Infected }\end{array}$ \\
\hline \multirow{4}{*}{$\begin{array}{c}\text { Gram positive bacteria } \\
(n=87) \\
43.72 \%\end{array}$} & Staphylococcus aureus & 74 & 37.2 \\
\hline & Streptococcus pyogenes & 6 & 3.0 \\
\hline & Streptococcus pneumonia & 5 & 2.5 \\
\hline & Enterococcus spp. & 2 & 1.0 \\
\hline \multirow{5}{*}{$\begin{array}{c}\text { Gram negative } \\
\text { bacteria } \\
(n=94) \\
47.24 \%\end{array}$} & Pseudomonas aeruginosa & 69 & 34.7 \\
\hline & Escherichia coli & 8 & 4.0 \\
\hline & Proteus mirabilis & 8 & 4.0 \\
\hline & Klebsiella pneumonia & 5 & 2.5 \\
\hline & Enterobacter aerogenes & 4 & 2.0 \\
\hline \multirow{3}{*}{$\begin{array}{l}\text { Fungal Isolates } \\
(n=18) 9.05 \%\end{array}$} & Aspergillus spp. & 10 & 5.0 \\
\hline & Candida spp. & 8 & 4.0 \\
\hline & Total & 199 & --- \\
\hline
\end{tabular}

Table.5 Antibiotic sensitivity patterns of Gram-negative bacteria from ear swabs

\begin{tabular}{|c|c|c|c|c|c|c|c|c|c|c|c|c|c|c|c|}
\hline \multirow[b]{2}{*}{ Antibiotic } & \multicolumn{3}{|c|}{$\begin{array}{c}\text { P.aeruginosa } \\
(n=69)\end{array}$} & \multicolumn{3}{|c|}{$\begin{array}{l}\text { E.coli } \\
(n=7)\end{array}$} & \multicolumn{3}{|c|}{ E. aerogenes $(n=4)$} & \multicolumn{3}{|c|}{ P.mirabilis $(n=6)$} & \multicolumn{3}{|c|}{$\begin{array}{c}\text { K.pneumoniae } \\
(n=4)\end{array}$} \\
\hline & $\mathbf{S \%}$ & $1 \%$ & $\mathbf{R} \%$ & $\mathrm{S \%}$ & $1 \%$ & $\mathbf{R} \%$ & $\mathbf{S \%}$ & $1 \%$ & $\mathbf{R} \%$ & $\mathbf{S \%}$ & $1 \%$ & $\mathbf{R} \%$ & $\mathbf{S \%}$ & $1 \%$ & $\mathbf{R} \%$ \\
\hline CXM (0) & - & - & - & 50.0 & 12.5 & 37.5 & - & - & - & 50.0 & 0.0 & 50.0 & 40.0 & 0.0 & 60.0 \\
\hline $\mathrm{CXM}(\mathrm{P})$ & - & - & - & 62.5 & 0.0 & 37.5 & - & - & - & 50.0 & 0.0 & 50.0 & 40.0 & 0.0 & 60.0 \\
\hline CFM & - & - & - & 62.5 & 12.5 & 25.0 & - & - & - & 25.0 & 0.0 & 75.0 & 40.0 & 0.0 & 60.0 \\
\hline CAZ & 97.1 & 0.0 & 2.9 & 75.0 & 0.0 & 25.0 & 100 & 0.0 & 0.0 & 75.0 & 0.0 & 25.0 & 60.0 & 0.0 & 40.0 \\
\hline CTR & - & - & - & 75.0 & 0.0 & 25.0 & 100 & 0.0 & 0.0 & 75.0 & 0.0 & 25.0 & 80.0 & 0.0 & 20.0 \\
\hline $\mathrm{CPM}$ & 97.1 & 0.0 & 2.9 & 75.0 & 0.0 & 25.0 & 100 & 0.0 & 0.0 & 75.0 & 0.0 & 25.0 & 80.0 & 0.0 & 20.0 \\
\hline $\mathrm{OF}$ & 81.2 & 2.9 & 15.9 & 87.5 & 0.0 & 12.5 & 100 & 0.0 & 0.0 & 75.0 & 12.5 & 12.5 & 100 & 0.0 & 0.0 \\
\hline CIP & 82.6 & 0.0 & 17.4 & 87.5 & 0.0 & 12.5 & 100 & 0.0 & 0.0 & 75.0 & 12.5 & 12.5 & 100 & 0.0 & 0.0 \\
\hline LE & 82.6 & 0.0 & 17.4 & 87.5 & 0.0 & 12.5 & 100 & 0.0 & 0.0 & 87.5 & 0.0 & 12.5 & 100 & 0.0 & 0.0 \\
\hline AMC & - & - & - & 75.0 & 0.0 & 25.0 & - & - & - & 100 & 0.0 & 0.0 & 80.0 & 0.0 & 20.0 \\
\hline PT & 100 & 0.0 & 0.0 & 100 & 0.0 & 0.0 & 100 & 0.0 & 0.0 & 100 & 0.0 & 0.0 & 100 & 0.0 & 0.0 \\
\hline GEN & 85.5 & 2.9 & 11.6 & 100 & 0.0 & 0.0 & 100 & 0.0 & 0.0 & 87.5 & 0.0 & 12.5 & 100 & 0.0 & 0.0 \\
\hline $\mathrm{AK}$ & 88.4 & 0.0 & 11.6 & 100 & 0.0 & 0.0 & 100 & 0.0 & 0.0 & 87.5 & 0.0 & 12.5 & 100 & 0.0 & 0.0 \\
\hline COT & - & - & - & 75.0 & 0.0 & 25.0 & 75.0 & 0.0 & 25.0 & 0.0 & 0.0 & 100 & 60.0 & 0.0 & 40.0 \\
\hline TE & - & - & - & 75.0 & 0.0 & 25.0 & 75.0 & 25.0 & 0.0 & - & - & - & 60.0 & 0.0 & 40.0 \\
\hline IPM & 100 & 0.0 & 0.0 & 100 & 0.0 & 0.0 & 100 & 0.0 & 0.0 & 100 & 0.0 & 0.0 & 100 & 0.0 & 0.0 \\
\hline AT & 85.5 & 10.1 & 4.3 & - & - & - & - & - & - & - & - & - & - & - & - \\
\hline TCC & 78.3 & 15.9 & 5.8 & - & - & - & - & - & - & - & - & - & - & - & - \\
\hline
\end{tabular}

CXM(O)- Cefuroxime (Oral), CXM (P)- Cefuroxime (Parentral), CFM- Cefixime, CAZ- Ceftazidime, CTR- Ceftriaxone, CPM-Cefepime, OF- Ofloxacin, CIP- Ciprofloxacin, LE- Levofloxacin, AMC- Amoxyclav-clavulanate, PT- Piperacillintazobactam, GEN- Gentamycin, AK- Amikacin, COT- Cotrimoxazole, TE- Tetracycline, IPM- Imipenem, AT- Aztreonam, TCC- Ticarcillin/Clavulanate.

$\mathrm{S}=$ Sensitive, R- Resistant, I= Intermediate 
Table.6 Antibiotic sensitivity patterns of Gram-positive bacteria from ear swabs

\begin{tabular}{|c|c|c|c|c|c|c|c|c|c|c|c|c|}
\hline \multirow[b]{2}{*}{ Antibiotic } & \multicolumn{3}{|c|}{ S.aureus $(n=74)$} & \multicolumn{3}{|c|}{ S.pyogenes $(n=6)$} & \multicolumn{3}{|c|}{$\begin{array}{c}\text { S.pneumoniae } \\
(n=5)\end{array}$} & \multicolumn{3}{|c|}{$\begin{array}{c}\text { Enterococcus spp. } \\
(n=2)\end{array}$} \\
\hline & $\mathrm{S} \%$ & $\mathbf{I \%}$ & $\mathbf{R} \%$ & $\mathrm{S \%}$ & $\mathbf{I \%}$ & $\mathbf{R} \%$ & S\% & $1 \%$ & $\mathbf{R} \%$ & $\mathrm{S \%}$ & $\mathbf{I \%}$ & $\mathbf{R} \%$ \\
\hline $\mathrm{P}$ & 6.8 & 0.0 & 93.2 & 100 & 0.0 & 0.0 & 100 & 0.0 & 0.0 & 50.0 & 0.0 & 50.0 \\
\hline AMP & - & - & - & 100 & 0.0 & 0.0 & - & - & - & 50.0 & 0.0 & 50.0 \\
\hline CTR & - & - & - & 100 & 0.0 & 0.0 & - & - & - & - & - & - \\
\hline $\mathrm{CPM}$ & - & - & - & 100 & 0.0 & 0.0 & - & - & - & - & - & - \\
\hline CIP & 25.7 & 14.9 & 59.5 & - & - & - & - & - & - & 50.0 & 0.0 & 50.0 \\
\hline OF & 25.7 & 14.9 & 59.5 & 66.7 & 0.0 & 33.3 & 100 & 0.0 & 0.0 & 50.0 & 0.0 & 50.0 \\
\hline LE & 40.5 & 33.8 & 25.7 & 66.7 & 0.0 & 33.3 & 100 & 0.0 & 0.0 & 50.0 & 0.0 & 50.0 \\
\hline GEN & 85.1 & 12.2 & 2.7 & - & - & - & - & - & - & - & - & - \\
\hline $\mathrm{AK}$ & 97.3 & 1.4 & 1.4 & - & - & - & - & - & - & - & - & - \\
\hline AZM & - & - & - & 50.0 & 0.0 & 50.0 & 80.0 & 0.0 & 20.0 & & & \\
\hline $\mathrm{E}$ & 68.9 & 9.5 & 21.6 & 50.0 & 0.0 & 50.0 & 80.0 & 0.0 & 20.0 & 50.0 & 0.0 & 50.0 \\
\hline $\mathrm{CD}$ & 78.4 & 0.0 & 21.6 & 66.7 & 0.0 & 33.3 & 80.0 & 0.0 & 20.0 & - & - & - \\
\hline $\mathrm{C}$ & 97.3 & 0.0 & 2.7 & 83.3 & 0.0 & 16.7 & 100 & 0.0 & 0.0 & 100 & 0.0 & 0.0 \\
\hline TE & 91.9 & 0.0 & 8.1 & 83.3 & 16.7 & 0.0 & 80.0 & 0.0 & 20.0 & 100 & 0.0 & 0.0 \\
\hline COT & 40.5 & 0.0 & 59.5 & - & - & - & 60.0 & 0.0 & 40.0 & - & - & - \\
\hline $\mathrm{LZ}$ & 100 & 0.0 & 0.0 & 100 & 0.0 & 0.0 & - & - & - & 100 & 0.0 & 0.0 \\
\hline TEI & 100 & 0.0 & 0.0 & - & - & - & - & - & - & 100 & 0.0 & 0.0 \\
\hline VA & 100 & 0.0 & 0.0 & 100 & 0.0 & 0.0 & 100 & 0.0 & 0.0 & 100 & 0.0 & 0.0 \\
\hline $\mathrm{CX}$ & 75.7 & 0.0 & 24.3 & - & - & - & - & - & - & - & - & - \\
\hline HLG & - & - & - & - & - & - & - & - & - & 100 & 0.0 & 0.0 \\
\hline
\end{tabular}

P- Penicillin, AMP- Ampicillin, CTR- Ceftriaxone, CPM- Cefepime, CIP-Ciprofloxacin, OF- Ofloxacin, LE- Levofloxacin, GEN- Gentamicin, AK- Amikacin, AZM- Azithromycin, E-Erythromycin, CDClindamycin, C- Chloramphenicol, TE- Tetracycline, COT- Cotrimoxazole, LZ- Linezolid, TEITeicoplanin, VA- Vancomycin, CX- Cefoxitin, HLG- High Level Gentamicin.

$\mathrm{S}=$ Sensitive, R- Resistant, I= Intermediate

In conclusion, the Bacterial ear infection is a foremost health trouble in the study area. S.aureus, $P$. aeruginosa, $P$. mirabilis and $E$. coli were the dominant isolates. In grampositive bacteria, high resistance was found in $S$. aureus isolates, penicillin (93.2\%) was found to be most resistant followed by ciprofloxacin, Ofloxacin, cotrimoxazole $(59.5 \%)$, and cefoxitin (24.3\%). Moreover, a considerable level of oxacillin-resistant S.aureus suggests the diffusion of Methicillin-resistant S.aureus in the community.
As the symptoms collapse, many patients stop taking antibiotics before the completion of therapy and let the partly resistant microbes to flourish. Patients should be instructed to evade such practice. Continuous and periodic assessment of microbiological pattern and antibiotic sensitivity of isolates is essential to decrease the potential risk of complications by early institution of appropriate treatment under expert supervision. I believe that data from this study may contribute to an effective management of ear infections. 


\section{Acknowledgements}

The study was done at Department of Microbiology \& Molecular Biology, Modern Diagnostic and Research Centre, Gurgaon. I would like to express my special appreciation and thanks to all the Patients whose samples were used in this study, my wife \& my father-mother who gives me inspiration to write the research papers, technicians of my department and our Director, Dr. D.S Yadav for his continuous guidance, constitutive criticism, and constant encouragement.

\section{References}

Alo, M.N., Anyimm, C. et al., 2012. Prevalence, antibiogram of bacterial pathogens associated with otitis media among primary school children in Ebonyi State. J. Pharm. Bio. Sci., 1(78): 17-20.

Arshad, M., Khan, N.U. et al., 2004. Sensitivity and spectrum of bacterial isolates in infectious otitis externa. $J$. Coll. Phy. Surg. Pak., 14: 146-149.

Aslam, M.A., Ahmed, Z. et al., 2004. Microbiology and drug sensitivity patterns of chronic suppurative otitis media. J. Coll. Physicians Surg. Pak., 14: 459-461.

Iqbal, B., J., Khan, W. et al., 2009. Frequency of chronic suppurative otitis media in the junior ranks of pak army. Pak. Armed forces Med. J., 59: 367-370.

Bardanis, J., Batzakakis, D., et al., 2003. Types and causes of otorrhea. Auris Naus Larynx, 30: 253-257.

Cheng, A.T., Youg, N.M. 1997. Inflammatory diseases of the ear. Indian J Pediatr 64: 747-753.

CLSI, Performance standards for antimicrobial susceptibility testing; Twenty-fifth International supplement., 2015. CLSI document
M100-S24. Wayne, PA: Clinical Laboratory and Standard Institute.

Grevers, G. 2010. Challenges in reducing the burden of otitis media disease: an ENT perspective on improving management andf prospects for prevention. Int. J. Pediatr. Otohinolaryngol., 74: 572-577.

Haraldsson, G., Holbrook, W.P. et al., 2004. Clonal similarity of salivary and nasopharyngeal Fusobacterium nucleatum in infants with acute otitis media experience. J. medical microbiol., 532: 165-5.

Haynes, D.S. 2002. Preoperative antibiotics in chronic supportive otitis media. Ear Nose Throat J., 81: 13-15.

Kristo, B., Buljan M. 2011. Microbiology of the chronic suppurative otitis media. Medicinki Glasnik, 8: 2.

Kumar, H., Seth., S. 2011. Bacterial and fungal study of 100 cases of chronic suppurative otitis media. J. Clin. Diagn. Res., 5(6); 1224-7.

Lodhi, M., Munir, T. et al., 2010. Chronic suppurative otitis media; Empiric quinolones in children. Professional Med. J., 17(3): 420-4.

Loy, A.H.C., Tan, A.L., et al., Microbiology of chronic suppurative otitis media in Singapore. Singapore Med. J., 43(6): 296-299.

Melaku, A., Lulseged, S., 1999. Chronic suppurative otitits media in childrens hospital in Addis, Ababa, Ethiopia. Ethiop Med. J., 37: 237-45.

Moorthy, P.N.S., Jadi, L. et al., 2013 Clinical application of microbiological study of chronic suppurative otitis media. Indian J. Otolaryngol. Head Neck Surg., 2: 290-94.

Mubassabi, K. 2007. Bacteriology of discharging ears. Ir. Med. J., 100: 379380.

Nazir, A., Kadri, S.M. 2014. Aerobic bacteriology of chronic suppurative 
otitis media: a hospital based study. Int. J. Res. Med. Sci., 2(4): 521-524.

Nwabuisi, C., Ologe F.E. 2002. Pathogenic agents of chronic suppurative otitis media in Ilorin, Nigeria. East Afr. Med. J., 79(4): 202-5.

Oguntibeju, O. 2003. Bacterial isolates from patients with ear infection. Indian J. Med. Microbiol., 21(4): 214-294.

Park, D.C., Lee, S.K. et al., 2008. Antimicrobial resistance of Staphylococcus from otorrhea in chronic suppurative otitis media and comparison with results of all isolated Staphylococci. Eur. J. Clin. Microbiol. Infec. Dis., 27(7); 571-7.

Poorey, V.K., Iyer, A. 2002. Study of bacterial flora in CSOM and its clinical significance. Indian $J$. Otolaryngol. Head Neck. Surg., 59: 91-95.

Prakash, M., Lakshmi, K. et al., 2013. Bacteriological profile and their antibiotic susceptibility pattern of cases of chronic suppurative otitis media. Asian J. Pharm. Clin. rRes., 6(3): 210-212.

Prakash, R., Juyal D. et al., 2013. Microbiology of chronic suppurative otitis media in a tertiary care setup of Uttarkhand State. India N. Am. J. Med. Sci., 5(4) :282-7.
Raakhee, T., and Unguturu, S.R. 2014. Bacteriological study of discharging ear in patients attending a tertiary care hospital. Int. J. Res. Med. Sci., 2(2): 602-606.

Richard, E.B., Roberts, M.K.1996. Otitis Media and its complications in Nelson's Textbook of pediatrics. 1814-1824.

Srivastava, A., Singh, R et al., 2011. Microbiological evaluation of an active tubotympanic type of chronic suppurative otitis media. Nepalese J. Ent, Head and Neck Surg., 12: 14-16.

Tobih, J.E., Taiwo, S.S. et al., 2006. Clinical and Microbiological profile of EAR INFECTIONJS IN Osogbo, Nigeria. Trop .Doct., 36: 165-166.

Wasihun, A.G., Zemene, Y. 2015. Bacterial profile and antmicrobial susceptibility patterns of otitis media in Ayder teaching and referral Hospital, Mekelle University, Northern Ethiopia. Springerplus, 4: 701.

World Health Organization. 1986. Prevention of deafness and hearing impairment. Geneva: report by the Director General. Document a29/14.

Yousuf, M., Majumder, K.A. 2011. Clinical study on chronic suppurative otitis media with cholesteatoma, Bangladesh. J. Otorhinolaryngol., 17(1): 42-7.

\section{How to cite this article:}

Rishabh Rajput. 2016. The spectrum of Different Pathogens causing Ear Infections and its Antimicrobial Sensitivity Patterns in Patients from Gurgaon, Haryana state, India. Int.J.Curr.Microbiol.App.Sci. 5(7): 343-352. doi: http://dx.doi.org/10.20546/ijcmas.2016.507.037 